\author{
Efrat Neter ${ }^{\star}$ Bat-El Levi and Sharon \\ Miller \\ Ruppin Academic Center, Israel \\ Dates: Received: 31 August, 2015; Accepted: 27 \\ October, 2015; Published: 29 October, 2015 \\ ${ }^{*}$ Corresponding author: Efrat Neter, Ruppin \\ Academic Center, Israel, Tel: +972-54-6462677; Fax: \\ +972-9-898-3043; E-mail: neter@ruppin.ac.il \\ www.peertechz.com \\ ISSN: 2455-5479 \\ Keywords: Compensatory health beliefs; \\ Vaccination; Mediation

\section{Research Article \\ My Child Doesn't Need to be Vaccinated Because We Lead a Healthy Lifestyle: Compensatory Health Beliefs Predicting Intentions and Reported Child Vaccination}

\section{Abstract}

Background: In the past two decades negative attitudes towards vaccination has surfaced among parents. These concerns are related to safety, adverse effects, lifestyle and religious issues. The present study focuses on compensatory health beliefs (CHBs), the beliefs that the adverse consequences of one health behavior are compensated by another health behavior, and examines whether CHB predicts behavior and mediates the association between the intention to vaccinate one's children and vaccination behavior.

Methods: Parents $(n=90)$ to children up to 6 years old filled an online questionnaire taping intentions and behaviors to vaccinate, self-efficacy to vaccinate, compensatory health beliefs related to vaccination and lifestyle.

Results: A significant negative association was found between $\mathrm{CHB}$ and intention to vaccinate and vaccination behavior $(r=-0.69, p<.01)$, and a non-significant positive association was found between $\mathrm{CHB}$ and lifestyle. $\mathrm{CHB}$ partially mediated the association between intention and vaccination behavior $\left(Z_{\text {Sobel }}=2.97, p<.01\right)$.

Conclusions: Future campaigns to increase vaccination should address $\mathrm{CHB}$ directly.

\section{Introduction}

Vaccinations are considered a major public health achievement, contributing to declines in morbidity and mortality from vaccinepreventable diseases [1]. Though childhood vaccination coverage in developed countries is high and stable [2], and is increasing (though less than the expected targets) in low and middle income countries [3], there are concerns over declining coverage in developed countries and resultant disease outbreaks, especially in lieu of the heated public debate on vaccination [4-8]. The public discourse, availed primarily through the Internet, and especially Web 2.0 participatory opportunities [9], sprang from variations between national policies in different countries, shifts in policy within the same country, and ongoing changes in vaccination protocols, creating a confusion among parents [2].

Reviews indicate that reasons for under-vaccination and nonvaccination are multiple [10], with vaccination decisions made "on a complex array of factors" along the decision process [4]. Parents' different concerns may translate into indifference, anti-vaccination convictions and hesitancy [2], all occurring in spite of an abundance of information [11]. Hesitant parents are of particular interest: they use reasoned arguments, balancing the risks and benefits of vaccination, and act accordingly, resulting in partial vaccination and intentional delay in vaccine administration to children [2].
These different responses are acknowledged by professionals who realize that current public health communications about vaccines may not be effective: the persuasive communication "may actually increase misperceptions or reduce vaccination intention" among some parents [12], specifically interventions aimed at increasing concerns about communicable diseases or correcting false claims that vaccines are counterproductive. This has led researchers to conclude that it is paramount to consider the opinions of laypeople [13], use multi-faceted approach [10] and to identify those who are particularly vulnerable to finding and using unreliable and misleading information [4].

\section{Compensatory health beliefs}

Novel approaches to understand and enhance vaccination uptake are tested. Such endeavors include developing decision aid tools [11], applying Fuzzy Trace theory [14] and compensatory health beliefs to the context of vaccination. The latter concept has been utilized in predicting influenza vaccination among adults [15].

Compensatory health beliefs $(\mathrm{CHB})$ are a self-defense strategy employed when people experience a mental conflict between cognitions and behaviors or between cognitions $[16,18]$. This mental conflict may arise because of giving into a harmful temptation (such as smoking) or failing to initiate an attractive long term goal (e.g., eating a balanced diet). The best way to reduce this cognitive dissonance would be to enact the preferred behavior, e.g., to quit 
smoking or eat healthy food, but as these behaviors are difficult to achieve, people opt for the next best option: compensate for the negative effects of the habit (smoking or over eating) by engaging in another heath behaviors such as exercising. CHBs allow people to maintain unhealthy behaviors because they ameliorate the negative feelings associated with these behavior and allow people to preserve their positive self-image.

$\mathrm{CHBs}$ have been applied in predicting influenza vaccination uptake among adults. Specifically, it was examined whether people who do not participate in vaccination hold CHBs that justify their non- adherence [15]. The excuse that was examined was leading a healthy lifestyle that compensates or exempts from the need to vaccinate. Indeed, leading a lifestyle of health and sustainability (LOHAS) was found to be related to a host of behaviors [19]. In the adults' flu vaccination study CHBs were found to be a negative mediator between intention and actual vaccination behavior, so that people with low intentions were more likely to believe themselves to be protected from influenza on account of their lifestyle and this was associated with decreased uptake of the flu shot. CHBs were viewed in this study as a post-decisional defense strategy but they could also operate in anticipation of engaging in a behavior and thus be employed by parents hesitant towards vaccinating their children [15].

The present study applied CHBs on lifestyle of health and sustainability (LOHAS) as an excuse to forgo child vaccination, that is the intention and (reported) behavior of either or not, and to what extent (partial or full) to vaccinate one's child. The study examined (1) whether people who live by LOHAS harbor more CHBs regarding vaccination, have less intention to vaccinate their children and report less vaccination; (2) whether people who harbor more CHBs have lowered intentions to vaccinate their children and report less uptake of the recommended vaccination regime; and (3) whether CHBs mediate the association between intentions and vaccination behavior, as found by the study on vaccinating oneself [15].

\section{Methods}

\section{Participants and procedure}

A total of 90 parents to children under 6 years old participated in the study. They included 69 women and 21 men, aged 25 and onward, with a mean age of $36.98(\mathrm{SD}=10.54)$. The average age of the men was higher $(M=41.81, S D=9.28)$ than the age of the women $(M=35.51$, $\mathrm{SD}=10.53)$. The modal educational attainment of participants was post-secondary $(31.9 \%)$ and $12.6 \%$ held advanced degrees.

Participants were recruited through social networks and Internet parenting forums. They filled an online anonymous questionnaire, providing background details and vaccination-related constructs.

\section{Measures}

Compensatory health beliefs: Compensatory health beliefs were assessed using an 8-item scale constructed by the authors, addressing different aspects of a healthy lifestyle such as eating organic food, exercise and stress avoidance. Participants responded to a 5-point Likert scale, ranging from 1 (strongly disagree) to 5 (strongly agree), Cronbach's alpha in the sample was 0.94 . Sample items are "we live in a healthy way and therefore no vaccination is needed; my children are relaxed and not stressed, therefore less in need of vaccination; organic food makes vaccination superfluous; we do a lot of physical exercise in our family and therefore our children do not need to vaccinate".

Intentions to vaccinate: Intentions to vaccinate were assessed using a 3-items scale from the Immunization Beliefs and Intention Measure [20]. Participants responded to a 5-point Likert scale, ranging from 1 (strongly disagree) to 5 (strongly agree), Cronbach's alpha in the present sample was 0.78 . Sample item is "I intend to take my child for vaccination".

Vaccination behavior: Vaccination behavior was assessed using a 3-items scale previously used in a health style survey [21]. Participants responded to a 5-point Likert scale, ranging from 1 (strongly disagree) to 5 (strongly agree), Cronbach's alpha in the present sample was 0.92 . Sample items are "my child has already received all recommended vaccines "and "my child has received some but not all recommended vaccines”.

Lifestyle: Lifestyle was assessed using items extracted from two questionnaires. Items $(n=19)$ on physical exercise, relaxation, eating and smoking were taken from the Determinants of Lifestyle Behavior Questionnaire [22]. Items were either on a 5-point Likert scale, ranging from 1 (strongly disagree) to 5 (strongly agree), or were on a semantic differential scale ranging from 0 (negative attitude) to 10 (positive attitude). Items related to environmental issues ( $\mathrm{n}=$ 8 items) were adapted from a study on environmental beliefs and intentions [17]. Items were on a 5-point Likert scale, ranging from 1 (does not characterize me) to 5 (characterizes me a lot). Sample items related to driving less by carpooling or taking public transportation; conserving paper by reading articles online and writing/editing papers on the computer instead of printing them out. All items were first standardized and then a mean was computed. Cronbach's alpha in the sample was 0.84 .

\section{Statistical analyses}

Descriptive analyses were first performed and Pearson correlations were calculated to examine the bivariate relations between measured variables. Regression analyses were performed to examine mediation (Hypotheses 3) with SPSS macros [23], specifically examining whether the effects of intention to vaccinate on vaccination behavior was mediated by compensatory health beliefs on lifestyle.

\section{Results}

\section{Descriptive results}

Descriptive statistics and Pearson correlations between the measured variables are presented in Table 1 . As can be seen, both intentions to vaccinate and reported vaccination behavior are moderate, reflecting hesitation in intentions and partial or delayed vaccination. In terms of the bivariate associations between lifestyle and intention, behavior and $\mathrm{CHB}$, no significant correlation emerged $(r=0.11, r=0.05, r=0.10$, respectively, all $p$ 's $>0.05)$.

The bivariate association between compensatory health beliefs and intentions to vaccinate was negatively significant $(r=-0.69, p<$ $0.01)$ as well as between compensatory health beliefs and vaccination behavior $(r=-0.69, p<0.01)$ so that the more compensatory health 
beliefs parents harbored, the less they intended to vaccinate their children and the less they have already carried out the vaccination schedule.

\section{CHB as mediator between intentions and vaccination behavior}

A mediation analysis quantifies the degree to which a predictor variables $\mathrm{X}$ (here: intention to vaccinate) is associated with the dependent variables $\mathrm{Y}$ (here: vaccination behavior) through a mediator variable $\mathrm{M}$ (here: compensatory health beliefs). Several paths are estimated: First, the path from X to $M$ (a), establishing that intentions to vaccinate are associated with compensatory health beliefs. Second, both the paths from X to $Y(c)$ and the path from $M$ to $\mathrm{Y}(\mathrm{b})$ are estimated. Finally, the model estimates the indirect path $\left(a^{*} b\right)$ of $X$ on $Y$ via M. Mediation is said to occur if this indirect path is significantly different from zero. Following current recommendations [23], we established this using bootstrapping $(5,000)$ resamples) to estimate a $95 \%$ confidence interval around the indirect path.

The indirect effect of intentions to vaccinate via compensatory health beliefs on vaccination behavior was significant (Table 2): the direct effect decreased when the indirect effect was taken into account, indicating partial mediation (Figure 1). Specifically, for every unit of increase in intentions to vaccinate, vaccination behavior increased by 0.81 units. Out of these, 0.17 were due to compensatory health beliefs $(\beta=.17,95 \%$ BCa CI: .05 - .36). Sobel test also indicated a significant

Table 1: Means, Standard Deviations, and Inter correlations between measured variables.

\begin{tabular}{|c|c|c|c|c|c|}
\hline Variables & M & SD & 2 & 3 & 4 \\
\hline Lifestyle & 0.00 & 0.67 & 0.11 & 0.05 & 0.10 \\
\hline Intentions to vaccinate & 3.69 & 1.34 & & $0.81^{\star *}$ & $-0.69 * *$ \\
\hline Vaccination Behavior & 3.84 & 1.41 & & & $-0.69 * *$ \\
\hline Compensatory health beliefs & 1.90 & 0.94 & & & \\
\hline
\end{tabular}

Table 2: Compensatory Health Beliefs Mediate the Effects of Intentions to Vaccinate on Vaccination Behavior.

\begin{tabular}{|c|c|c|c|c|c|c|c|}
\hline \multicolumn{2}{|c|}{} & $\begin{array}{l}\text { Total } \\
\text { effect }\end{array}$ & Direct effect & \multicolumn{3}{c|}{ Indirect effect } \\
\hline $\mathrm{a}(\mathrm{SE})$ & $\mathrm{b}(\mathrm{SE})$ & $\mathrm{c}(\mathrm{SE})$ & $\mathrm{c}^{\prime}(\mathrm{SE})$ & $\mathrm{a}{ }^{*} \mathrm{~b}$ & $\mathrm{SE}$ & $\begin{array}{c}\text { Lower } \\
95 \% \mathrm{Cl}\end{array}$ & $\begin{array}{c}\text { Upper } \\
95 \% \mathrm{Cl}\end{array}$ \\
\hline$-.69(.07)$ & $-.25(.08)$ & $.81(.06)$ & $.64(.08)$ & .17 & .08 & .05 & .36 \\
\hline
\end{tabular}

Note: ${ }^{*} p<.05 ;{ }^{* *} p<.01 ; \quad{ }^{* * *} p<.001 . \quad a-$ effect of independent variable on mediator, $b$ - effect of mediator on outcome variable, $c$ - total effect of independent variable on outcome variable.

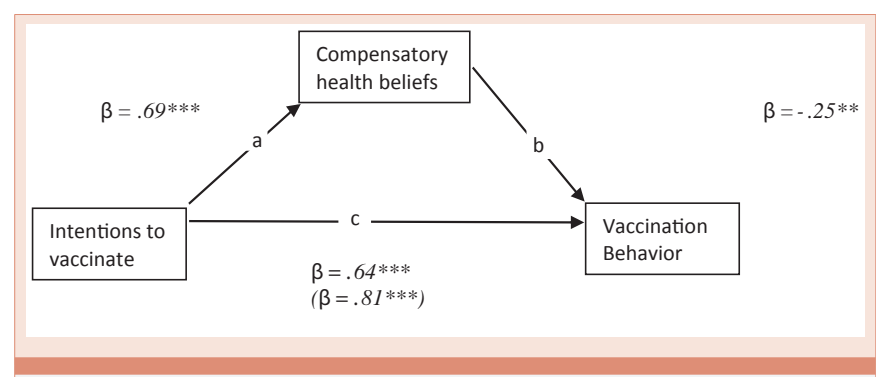

Figure 1: Mediating model of the effect of compensatory health beliefs on the association between intentions to vaccinate and vaccination behavior. Total effect is shown in frames.

${ }^{*} \mathrm{p}<.05, \quad{ }^{* *} \mathrm{p}<.01,{ }^{* * *} \mathrm{p}<.001$ mediation $\left(\mathrm{Z}_{\text {Sobel }}=2.97, \mathrm{p}<.01\right)$, and both $\mathrm{CHBs}$ and intentions to vaccinate explained $69 \%$ of the variance in vaccination behavior.

\section{Discussion}

This study set out to examine the contribution of $\mathrm{CHBs}$ in vaccinating children under 6 years old. It showed that the effects of intentions to vaccinate on vaccination behavior are mediated by CHBs. It also indicated that leading a healthy lifestyle in itself is not associated with either intentions to vaccinate, vaccination behavior or CHBs regarding a healthy lifestyle as exempting from vaccination.

As expected, intentions to vaccinate one's child were found to be related to vaccination behavior and, as predicted, this relationship was mediated by CHBs. Apparently, as parents hesitate about vaccination, they also reason with oneself about the advantages and risks regarding vaccinating their children; $\mathrm{CHBs}$ may be part of this deliberation process, as indicated by the partial (and not complete) mediation. Specifically, parents who view their lifestyle as a reason or an excuse not to vaccinate their children, complete the vaccination schedule or undertake vaccination to a lesser extent.

The current research advances the literature on $\mathrm{CHBs}$ and vaccination in three main ways. First, it replicates results on adult flu vaccination: $\mathrm{CHBs}$ were found to be negatively associated with both intentions and behaviors and mediate between the two [15], unlike findings on smoking [18], where CHBs were found to be associated with intentions to quit smoking but not with the behavior of quitting to smoke. Second, the study extended the findings from adults opting (or not) to vaccinate themselves to vaccinating (or not, or partially) one's children. The fact that a similar finding was recorded in two different countries may indicate that the phenomenon is present and may be spreading as an internal mental mechanism supporting the public discourse on non-adherence to vaccination. Third, CHBs were measured by a single item in the previous study on vaccination and CHBs [15], whereas the construct was measured by 8 items exhibiting high internal consistency in the current study. This feature constitute a strength of the present study.

Current research has important practical implications for public health. As adherence to vaccination is hotly contested among some parents in the Internet arena and traditional interventions do not yield the expected outcome of increased positive attitudes/ intentions and subsequent vaccination behavior [12], CHBs are a possible psychological mechanism involved in vaccination nonadherence: they explains how, in spite of all the 'correct' information, people decline adherence yet still see themselves as competent and responsible parents. The repeated documentation of the operation of $\mathrm{CHBs}$ on vaccination calls for interventions specifically targeting CHBs.

It should be noted that lifestyle of health and sustainability was not associated with either intentions, vaccination behavior or $\mathrm{CHBs}$ on healthy living as an excuse not to vaccinate. Though health behaviors tend to cluster, and especially cognitions around the health behaviors [24], this was not the case here, indicating that leading a healthy lifestyle and using it as an excuse for non-vaccination are two distinctly separate things. 
There are some limitations that need to be taken into account when interpreting the results of this study. First, the study relied exclusively on self-report data which poses limits on the conclusions that can be drawn from this research. Indeed, CHBs and intentions are inherently self-reported, but objective measures of vaccination behavior (i.e., medical or nursing records) are called for in order to establish the relationships among intentions, $\mathrm{CHBs}$ and vaccination behavior. Second, given the cross-sectional design of the study, inferences regarding causal relationships between the variables are precluded. Lastly, the sample in this study was small $(\mathrm{n}=90)$ and relatively well educated, with more than $40 \%$ of the sample with a university degree, possibly reflecting the recruitment method of using parenting Internet forums. Clearly, replication on a larger sample with a wider range of socioeconomic backgrounds is called for, since using the Internet for health purposes also depends on socioeconomic resources (Neter \& Brainin, 2012) and much of the discourse on vaccination is carried on using the Internet.

Despite these limitations, and especially considering the growing challenge of sporadic disease outbreaks due to preventable declining coverage, future research should reach out to wider segments of society, thus exploring other possible $\mathrm{CHBs}$ in predicting vaccination behavior. Future research should aspire to employ objective measures of vaccination behavior, and position the CHBs within a major health behavior theory such as Health Action Process Approach [25]. The latter will afford to discern CHBs operating in both the pre-intention motivational and post-intention volitional phases of the model, allowing better understanding of the $\mathrm{CHBs}$ and tailoring of messages by CHBs. Clearly, the next challenge is going beyond prediction of vaccination using $\mathrm{CHBs}$ into interventions that address the $\mathrm{CHBs}$ of the public.

\section{References}

1. Bloom DE, Canning D, Weston M (2005) The value of vaccination. World Economics 6: 15-39.

2. Velan B (2011) Acceptance on the move: public reaction to shifting vaccination realities. Hum Vaccin 7: 1261-1270.

3. World Health Organization (2014) [Internet]. Immunization surveillance, assessment and monitoring: vaccine preventable diseases.

4. Betsch C, Brewer NT, Brocard P, Davies P, Gaissmaier W, et al. (2012) Opportunities and challenges of Web 2.0 for vaccination decisions. Vaccine 30 3727-3733.

5. Covolo L, Mascaretti S, Caruana A, Orizio G, Caimi L, et al. (2013) How has the flu virus infected the Web? 2010 influenza and vaccine information available on the Internet. BMC Public Health 13: 83.

6. Kata A (2010) A postmodern Pandora's box: anti-vaccination misinformation on the Internet. Vaccine 28: 1709-1716.

7. Kata A (2012) Anti-vaccine activists, Web 2.0, and the postmodern paradigmAn overview of tactics and tropes used online by the anti-vaccination movement. Vaccine 30: 3778-3789.

8. McRee AL, Reiter PL, Brewer NT (2012). Parents' Internet use for information about HPV vaccine. Vaccine 30: 3757-3762.

9. Witteman HO, Zikmund-Fisher BJ (2012) The defining characteristics of Web 2.0 and their potential influence in the online vaccination debate. Vaccine 30 : 3734-3740.

10. Rainey JJ, Watkins M, Ryman TK, Sandhu P, Bo A, et al. (2011) Reasons related to non-vaccination and under-vaccination of children in low and middle income countries: findings from a systematic review of the published literature, 1999-2009. Vaccine 29: 8215-8221.

11. Wallace C, Leask J, Trevena LJ (2006) Effects of a web based decision aid on parental attitudes to MMR vaccination: a before and after study. BMJ 332:146-149.

12. Nyhan B, Reifler J, Richey S, Freed GL (2014) Effective messages in vaccine promotion: a randomized trial. Pediatrics 133: e835-e842.

13. Velan B, Kaplan G, Ziv A, Boyko V, Lerner-Geva L (2011) Major motives in non-acceptance of $\mathrm{A} / \mathrm{H} 1 \mathrm{~N} 1$ flu vaccination: the weight of rational assessment. Vaccine 29: 1173-1179.

14. Reyna VF (2012) Risk perception and communication in vaccination decisions: a fuzzy-trace theory approach. Vaccine 30: 3790-3797.

15. Ernsting A, Schwarzer R, Lippke S, Schneider M (2012) "I Don't Need a Flu Shot Because I Lead a Healthy Lifestyle": Compensatory Health Beliefs Make Vaccination Less Likely. J Health Psychol 18: 825-836.

16. Knäuper B, Rabiau M, Cohen O, Patriciu N (2004) Compensatory health beliefs: Scale development and psychometric properties. Psychology \& Health 19: 607-624

17. Radtke T, Scholz U, Keller R, Hornung R (2012) Smoking is ok as long as I eat healthily: Compensatory Health Beliefs and their role for intentions and smoking within the Health Action Process Approach. Psychology \& Health 27: 91-107.

18. Kim MJ, Lee CK, Gon Kim W, Kim JM (2013) Relationships between lifestyle of health and sustainability and healthy food choices for seniors. International Journal of Contemporary Hospitality Management 25: 558-576.

19. Tickner S, Leman PJ, Woodcock A (2010) The Immunisation Beliefs and Intentions Measure (IBIM): predicting parents' intentions to immunise preschool children. Vaccine 28: 3350-3362.

20. Kennedy A, Basket M, Sheedy K (2011) Vaccine attitudes, concerns, and information sources reported by parents of young children: results from the 2009 Health Styles survey. Pediatrics 127: S92-S99.

21. Lakerveld J, Bot SD, Chinapaw MJ, Knol DL, de Vet HC, et al. (2011) Measuring pathways towards a healthier lifestyle in the Hoorn Prevention Study: the Determinants of Lifestyle Behavior Questionnaire (DLBQ). Patient Education and Counseling 85: e53-e58.

22. Toner K, Gan M, Leary MR (2014) The impact of individual and group feedback on environmental intentions and self-beliefs. Environment and Behavior 46: 24-45

23. Hayes AF (2013) Introduction to mediation, moderation, and conditional process analysis: A regression-based approach. New York and London: Guilford Press.

24. Fleig L, Ngo J, Roman B, Ntzani E, Satta P, et al. (2015) Beyond single behaviour theory: Adding cross behaviour cognitions to the health action process approach. Br J Health Psychol 20: 824-841.

25. Schwarzer R (2008) Modeling health behavior change: How to predict and modify the adoption and maintenance of health behaviors. Applied Psychology 57: 1-29. 